\title{
Somatotopic Activation of Opioid Systems by Target-Directed Expectations of Analgesia
}

\author{
Fabrizio Benedetti, Claudia Arduino, and Martina Amanzio \\ Department of Neuroscience and Center for the Neurophysiology of Pain, University of Torino Medical School, \\ 10125 Torino, Italy
}

\begin{abstract}
We induced specific expectations of analgesia on four different parts of the body to understand how endogenous opioid systems are activated by expectancies. The left hand, right hand, left foot, and right foot were simultaneously stimulated by means of a subcutaneous injection of capsaicin, which produces a painful burning sensation. Specific expectations of analgesia were induced by applying a placebo cream on one of these body parts and by telling the subjects that it was a powerful local anesthetic. In such a way, expectancy of the anesthetic effect was directed only toward the part on which the placebo cream was applied. We found that a placebo analgesic response occurred only on the treated part, whereas no variation in pain sensitivity was found on the untreated parts. If the same experiment was performed after an intravenous infusion
\end{abstract}

Several lines of evidence indicate that some types of placebo activate endogenous opioid systems (Levine et al., 1978; Grevert et al., 1983; Levine and Gordon, 1984; Benedetti et al., 1995; Benedetti, 1996; Benedetti and Amanzio, 1997), although nonopioid mechanisms can play an important role in some situations (Gracely et al., 1983; Grevert et al., 1983). Fields and Levine (1984) analyzed the different psychological and/or environmental circumstances that might determine whether the placebo response has an opioid component. Recently, we have elucidated, at least in part, some of these circumstances (Amanzio and Benedetti, 1999). In fact, in accordance with previous studies (Fields and Levine, 1984; Voudouris et al., 1990; Fields and Price, 1997; Montgomery and Kirsch, 1997; Price and Fields, 1997), we showed that a placebo analgesic response can be elicited by expectation cues and/or conditioning. We found that expectationinduced placebo analgesia was associated with opioid activation, whereas conditioning-induced placebo analgesia was mediated by specific neurochemical pathways, depending on the drug used for conditioning (Amanzio and Benedetti, 1999).

Although these studies clarify some of the circumstances necessary for the activation of opioid systems, we do not know how these endogenous opioids are released. In other words, the problem is to understand how expectancies activate endogenous opioids and where the expectation-activated opioids are released within the CNS. Are they released throughout the brain, thus affecting the entire body, or rather do they show a high-order

\footnotetext{
Received Nov. 18, 1998; revised Feb. 1, 1999; accepted Feb. 16, 1999.

This work was supported by grants from Ministero dell'Università e della Ricerca Scientifica e Tecnologica and Consiglio Nazionale delle Ricerche Coordinate Project on Trigeminal Pain.

Correspondence should be addressed to Fabrizio Benedetti, Dipartimento di Neuroscienze, Università di Torino, Corso Raffaello 30, 10125 Torino, Italy. Copyright (C) 1999 Society for Neuroscience $\quad 0270-6474 / 99 / 193639-10 \$ 05.00 / 0$
}

of the opioid antagonist naloxone, this highly spatial-specific placebo response was totally abolished, indicating that it was completely mediated by endogenous opioid systems. These findings show that a spatially directed expectation of pain reduction is capable of inducing a specific effect only on the part of the body which is the target of the expectation. Most important, this specific effect is mediated by endogenous opioids, indicating that placebo-activated opioids do not act on the entire body but only on the part where expectancy is directed. This suggests that a highly organized and somatotopic network of endogenous opioids links expectation, attention, and body schema.

Key words: analgesia; pain; capsaicin; placebo; opioid systems; expectation; attention; body schema organization? At least two possibilities can be envisaged. First, endogenous opioids could act as modulators and/or hormones throughout the nervous system, thus affecting pain sensitivity and responsiveness of the entire body. Second, the activated opioids could act at a more local level, for instance, as mediators or transmitters of a specific neuronal circuitry. In this second case, we should expect no change of pain sensitivity on the entire body. Interestingly, Montgomery and Kirsch (1996) showed that specific expectancies may produce specific placebo responses. These authors found that some types of placebo analgesia cannot be explained by mechanisms that would affect the entire body (e.g., release of opioids throughout the nervous system). In fact, by administering a placebo in the guise of a local anesthetic on one part of the body, there were no changes in pain responsiveness on the other parts.

Taking into account these considerations, the present study was aimed at analyzing (1) whether endogenous opioids are activated by specific expectations, such as the expectancy that the left hand, but not other parts of the body, will be less sensitive to pain, and (2) whether the activated endogenous opioids affect only the part of the body where the expectation is directed or, rather, the entire body. In such a way, we could understand whether the placeboactivated opioids act throughout the nervous system or are confined to specific neuronal networks.

\section{MATERIALS AND METHODS}

Subjects. A total of 173 subjects participated in the study after they signed a written informed consent in which the experimental procedure was described and the use of subcutaneous capsaicin and intravenous naloxone was explained in detail. Each subject underwent a clinical examination in which the electrocardiogram was recorded. All subjects with heart problems were eliminated from the study. Many subjects reported a 


\begin{tabular}{|c|c|c|c|}
\hline & $\begin{array}{l}\text { Subjects } \\
\text { (males/females) }\end{array}$ & Age & Weight \\
\hline Group 1 (natural history) & $48(26 / 22)$ & $38.2 \pm 8.5$ & $63.4 \pm 10.8$ \\
\hline $\begin{array}{l}\text { Group } 2 \text { (hidden nalox- } \\
\text { one) }\end{array}$ & $20(10 / 10)$ & $36.1 \pm 7.6$ & $63.8 \pm 7.8$ \\
\hline $\begin{array}{l}\text { Group } 3 \text { (placebo on left } \\
\text { hand) }\end{array}$ & $25(13 / 12)$ & $40.4 \pm 9.8$ & $59.7 \pm 9.5$ \\
\hline $\begin{array}{l}\text { Group } 4 \text { (placebo on left } \\
\text { hand plus naloxone) }\end{array}$ & $27(13 / 14)$ & $36.7 \pm 8.8$ & $60.1 \pm 11.4$ \\
\hline $\begin{array}{l}\text { Group } 5 \text { (placebo on right } \\
\text { hand and left foot) }\end{array}$ & $24(12 / 12)$ & $39.0 \pm 7.6$ & $65.2 \pm 11.0$ \\
\hline $\begin{array}{l}\text { Group } 6 \text { (placebo on right } \\
\text { hand and left foot plus } \\
\text { naloxone) }\end{array}$ & $29(16 / 13)$ & $37.5 \pm 9.1$ & $61.9 \pm 8.4$ \\
\hline
\end{tabular}

previous experience with analgesics, both opioids and nonopioids, for different types of pathological conditions, such as headache and surgery. All experimental procedures were conducted in conformance with the policies and principles contained in the Declaration of Helsinki. The 173 subjects were subdivided into six groups, whose characteristics are shown in Table 1 . The ratio of males/females, age, and weight did not differ among the different groups.

Pain stimuli. Pain was induced experimentally by means of a subcutaneous injection of $10 \mu \mathrm{g}$ of capsaicin (8-methyl $N$-vanillyl 6-nonamide) (Fluka, Neu-Ulm, Germany) in $10 \mu \mathrm{l}$ of a polyoxyethylene(20)sorbetan monooleate (Tween 80) saline vehicle. The injection of $10 \mu \mathrm{g}$ of capsaicin produces a painful burning sensation reaching a peak within the first minute and lasting 9-12 min (LaMotte et al., 1991). The capsaicin was injected simultaneously into the dorsal side of the left hand, right hand, left foot, and right foot. To do this, a 30 gauge needle was inserted subcutaneously and connected to a catheter, which, in turn, was connected to an infusion pump (Fig. 1). The needles and the catheters were filled previously with the capsaicin solution. However, to avoid capsaicin emission during the subcutaneous insertion of the needle, the needle was filled only partially, leaving $10 \mu \mathrm{l}$ of empty space in proximity to the tip. Simultaneous injection of capsaicin under the skin of the hands and feet was assured by the infusion pump, which was programmed to deliver $20 \mu \mathrm{l}(10 \mu \mathrm{l}$ of the empty part of the needle plus $10 \mu \mathrm{l}$ of the filled part). In such a way, the injection of the $10 \mu \mathrm{l}$ of capsaicin was performed in $\sim 500 \mathrm{msec}$

Experimental design. The experiments were performed according to a randomized double-blind design in which neither the subject nor the experimenter knew what drug was administered. This was true for both naloxone and the local anesthetic (see below), so that we were completely blind in all experimental procedures. The subjects reclined on a bed, and a needle was inserted into a vein of the right arm (Fig. 1). The needle was connected to a line, $1 \mathrm{~m}$ long, through which a slow infusion of $5 \%$ glucose solution was administered. The intravenous line reached a screen behind the subject's bed, so that hidden injections could be performed by the experimenter.

The following procedure was used in all 173 subjects. After the simultaneous injection of capsaicin into the hands and feet, the subjects had to judge the time course of pain on these four body parts for the first $15 \mathrm{~min}$. To do this, four silver-chloride electrodes were positioned on the two wrists and the two ankles (Fig. 1) and connected to a stimulator and a constant current unit, thus avoiding the variability of skin-electrode impedance and warranting constant electrical stimuli throughout the experiment. Starting from $21 \mathrm{sec}$ before each minute, a mild electric shock was delivered every $7 \mathrm{sec}$ sequentially on the left hand, right hand, left foot, and right foot (Fig. 2). These stimuli were used to signal on which part of the body the capsaicin pain had to be judged. For example, when the shock was delivered to the left hand, the subjects had to judge the capsaicin burning sensation on the left hand, when the shock was delivered to the right foot they had to judge capsaicin pain on the right foot, and so forth. Both hands and both feet were tested for all the first 15 min after capsaicin injection (Fig. 2). The order of stimulation on the hands and feet was changed at random every minute. Pain intensity was judged according to a numerical rating scale (NRS), ranging from 0 indicating no pain to 10 indicating unbearable pain.

The data from every subject were stored in a computer according to a polar coordinate system subdivided into four quadrants. The upper left quadrant represented the left hand, the upper right the right hand, the lower left the left foot, and the lower right the right foot (Fig. 3). The angular coordinates represented the time (in minutes) from capsaicin injection and, because each quadrant had an amplitude of $90^{\circ}$ and the pain course was analyzed for $15 \mathrm{~min}$, each minute had an amplitude of $6^{\circ}$. The distance from the center of the polar system represented the pain intensity according to the NRS scores. An example taken from three different subjects is shown in Figure 3 (see the details in Results). For instance, the pain course of the right hand of the subject in Figure $3 A$ was represented by a decrease of pain intensity (decrease of the distance from the center), starting from the first through the fifteenth minute (from 0 through $90^{\circ}$ ).

Although this procedure was used in all subjects, they received different treatments before capsaicin injection (Fig. 2). Group 1 received a hidden intravenous injection of saline $(\mathrm{NaCl} 0.9 \%) 15$ min before the subcutaneous injection of capsaicin (natural history group). Group 2 received a hidden intravenous injection of naloxone (Crinos, Como, Italy) at a dose of $0.14 \mathrm{mg} / \mathrm{kg}$ in sterile solution of $\mathrm{NaCl} 0.9 \%$ (infusion rate, $0.1 \mathrm{ml} / \mathrm{sec}$; total inf usion time, ranging from 180 to $240 \mathrm{sec}$ ) (hidden naloxone group). This group was used to assess whether naloxone per se affected the burning pain sensation induced by capsaicin. In Groups 1 and 2, naloxone and saline were administered according to the doubleblind design.

In Group 3, a neutral cream (a mixture of oil of thyme and water) was applied around the subcutaneous needle of the left hand $10 \mathrm{~min}$ before capsaicin injection (Fig. 2). These subjects were told that the cream was a potent local anesthetic reducing the burning sensation of capsaicin. A hidden intravenous saline injection was performed $15 \mathrm{~min}$ before the injection of capsaicin. Group 4 received the same treatment as Group 3, but a hidden intravenous injection of naloxone $(0.14 \mathrm{mg} / \mathrm{kg})$ was performed $15 \mathrm{~min}$ before the capsaicin injection (Fig. 2). Groups 5 and 6 were treated as groups 3 and 4; however, the placebo cream was applied to the right hand and left foot (Fig. 2). To apply the neutral cream according to a double-blind design, in 20 additional subjects (five for each group), we applied a lidocaine $5 \%$ cream, which really anesthetized the skin around the subcutaneous needle.

Assessment of pain thresholds and allodynia. Beside the time course of capsaicin pain, we also assessed the variations of pain threshold in the capsaicin-induced allodynia. To do this, we used electrical stimulation. At the end of the experimental procedure described previously, the subcutaneous needles were taken out, and the four electrodes were positioned on the skin where capsaicin had been injected. The diameter of the electrodes was $6 \mathrm{~mm}$. Pain thresholds on the hands and the feet were assessed at 30 and $120 \mathrm{~min}$ after capsaicin injection. To do this, the stimulator delivered one stimulus per second, and the subjects had to adjust the stimulus intensity by using a hand-grip, until a painful electrical stimulus was perceived. It should be remembered that the electrical stimuli were delivered through a constant current unit, thus avoiding the variability of skin-electrode impedance. In such a way, pain thresholds were expressed in milliamperes. The subjects assessed their pain thresholds sequentially on the four parts of the body, and the sequence of pain threshold assessments on both hands and both feet was changed at random in each subject and each group. These pain thresholds were compared with the normal thresholds, which had been measured before starting the experiment and before inserting the subcutaneous needles. The variations of pain threshold in capsaicin-induced allodynia were expressed as the percentages of the threshold of the preinjection controls (see Fig. 7).

Statistical analysis. The differences between and within treatments were tested by means of the ANOVA, followed by the Newman-Keuls multiple range test for multiple comparisons. The data from single subjects were transformed from the polar coordinate system to the classic cartesian coordinates to analyze means and SDs of a single group. In addition, the response of each subject was expressed as the area under the curve, which was calculated according to the triangulation method of the study by Winter and Flataker (1949). The data are presented as mean \pm SD. Differences were considered to be statistically significant at $p<0.05$. 


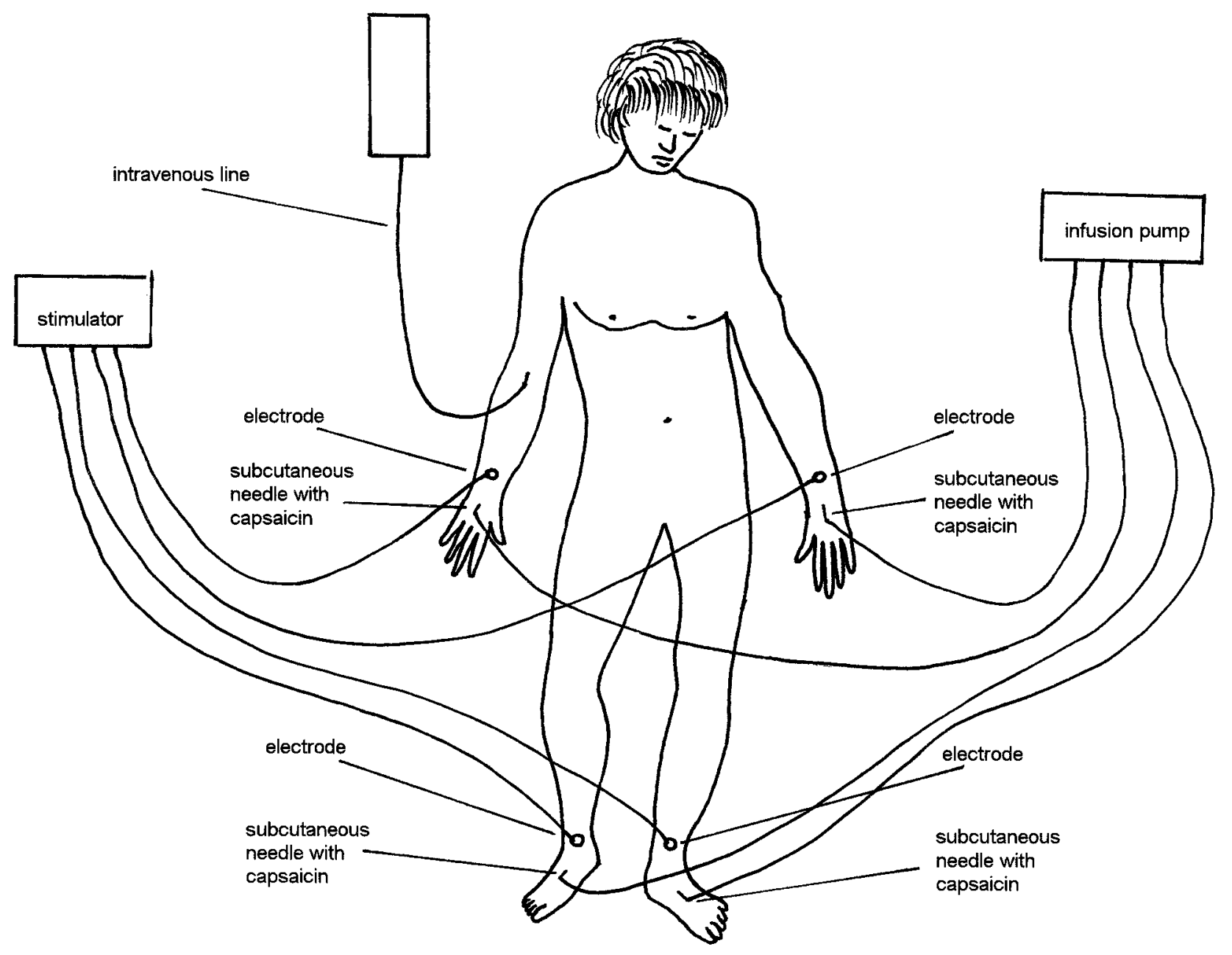

Figure 1. Experimental setup. Four subcutaneous needles, which are filled with capsaicin, are inserted under the skin of the dorsal side of both hands and both feet and connected to an infusion pump. The four electrodes are used to signal on which part of the body the subjects had to focus their attention and to judge capsaicin pain intensity. Naloxone was administered through the intravenous line.

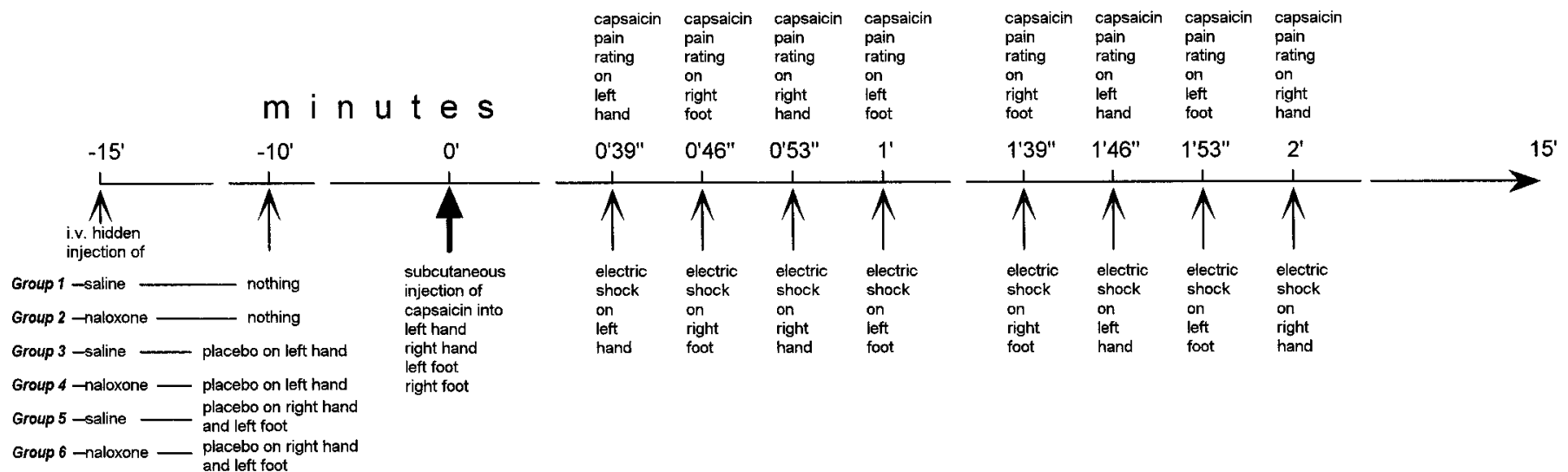

Figure 2. Experimental design. Starting from time 0, the same design was used for all 173 subjects. In contrast, they were subdivided into six groups that received different treatments at 15 and 10 min before time 0 .

\section{RESULTS}

\section{Analysis of the data from single subjects}

To see the outcome of a single subject at a glance during the experiment, the polar coordinate system was displayed on the computer screen. In such a way, the results for a single subject could be inspected in real time. Figure 3 shows three examples taken from three representative subjects; each polar system was taken directly from the computer screen. The subject in $A$ belongs to the natural history group and shows a pain time course that is equal on both hands and both feet. The subject in $B$ received the 


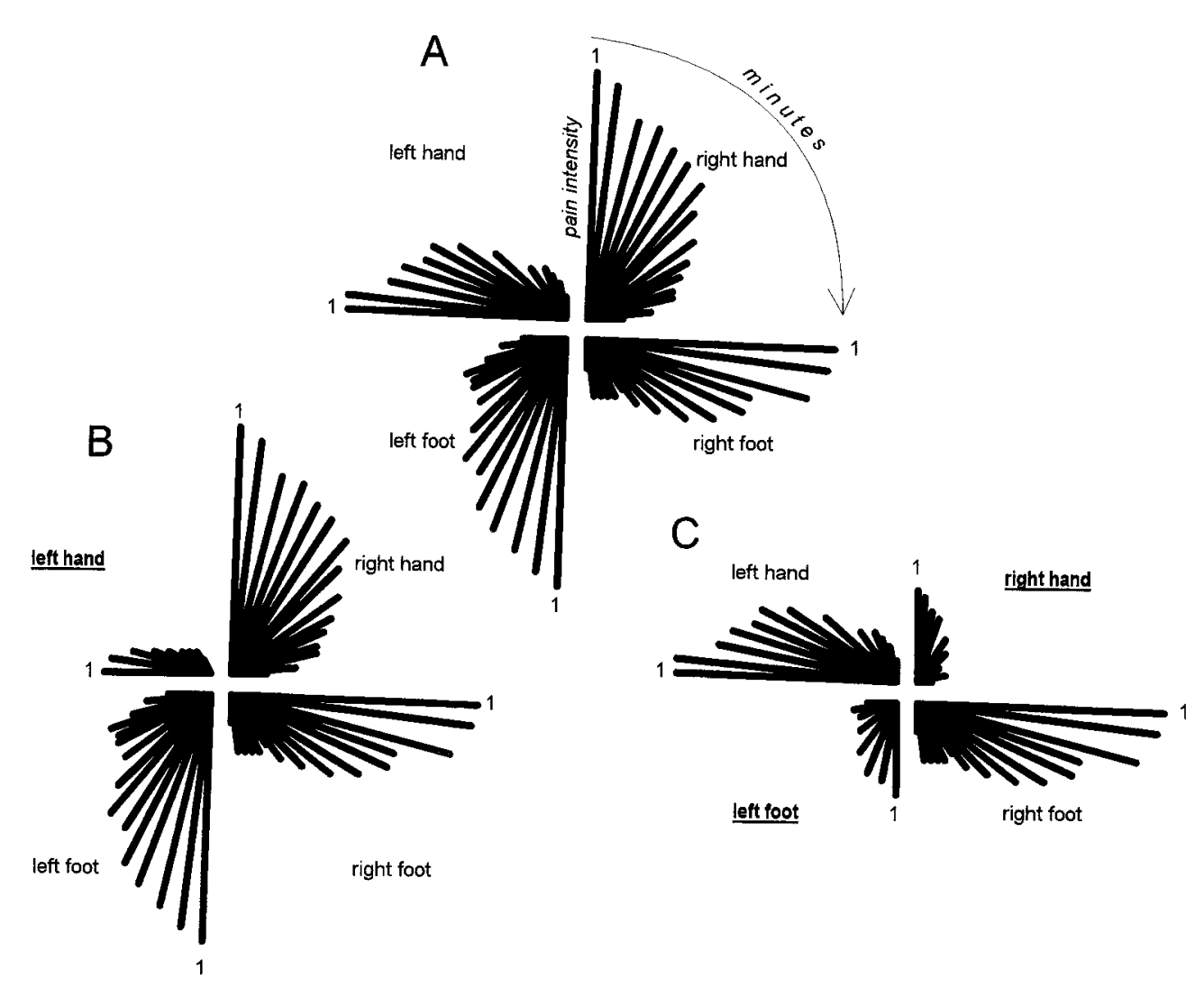

Figure 3. Data from three representative subjects in polar coordinate systems. The subject in $A$ belongs to the natural history group; thus, he did not receive any treatment. The four quadrants represent the four different parts of the body. For each quadrant, the pain time course is shown in which the distance from the center represents pain intensity and the angular coordinates represent the minutes after capsaicin injection. The first minute after capsaicin injection is shown for each quadrant. The subject in $B$ was treated with a placebo cream on the left hand (underlined), so that expectation of analgesia was directed to the left hand. Accordingly, a placebo pain reduction occurred in $C$ was treated with a placebo cream on the right hand and left foot (underlined), so that expectation of analgesia was directed to the right hand and left foot. Accordingly, a placebo pain reduction occurred specifically on the right hand and left foot. specifically on the left hand. The subject

placebo cream on the left hand, so that a reduction of pain was found specifically on the left hand. The subject in $C$ received the placebo cream on the right hand and the left foot, so that pain reduction occurred specifically on the right hand and left foot.

The following data and statistical analyses within and between groups were obtained by transforming the polar coordinate systems of all subjects into cartesian coordinates.

\section{The natural history of capsaicin pain}

The natural history of the pain induced by capsaicin (group 1) is shown in Figure $4 A$ for both hands and both feet. The statistical analysis of the areas under the curves is shown in Table 2 in which it can be noted that the ANOVA on the right represents the analysis within a single group, whereas the ANOVA at the bottom represents the analysis between the two groups for both the hands and the feet. It can be seen that no significant difference in pain time course was found between the four parts of the body, as shown by the analysis within group 1 of Table 2 . The consistency of the four pain time courses in the same group represents an important starting point to unravel possible differences on hands and feet.

The natural history was not modified by an intravenous dose of $0.14 \mathrm{mg} / \mathrm{kg}$ naloxone (group 2), indicating that naloxone per se did not affect this type of experimental pain. Figure $4 B$ shows that the hidden naloxone did not produce differences compared with the natural history. This is better evidenced by the statistical analysis of Table 2 in which no significant differences were found within group 2 and between groups 1 and 2 .

\section{Hand-directed expectancy of analgesia}

When the placebo cream was applied to the left hand, expectation of analgesia was specifically directed to the left hand (group 3). In this case, we observed a clear-cut placebo effect only on the left hand, whereas the other hand and the feet were unaffected (Fig. $5 A$ ). In fact, the response to capsaicin of the left hand, expressed as the area under the curve, was significantly smaller than the natural history $\left(F_{(1,71)}=35.5 ; p<0.001\right)$. In contrast, no difference between group 3 and the natural history was found for the right hand $\left(F_{(1,71)}=2.57 ; p=0.114\right)$, left foot $\left(F_{(1,71)}=1.94 ; p=\right.$ $0.168)$, and right foot $\left(F_{(1,71)}=0.48 ; p=0.492\right)$. Table 3 shows the analysis within group 3; it can be seen that the ANOVA on the right and the Newman-Keuls multiple range test ( $q$ coefficient) indicate a significant difference between the left hand and the other parts of the body.

If the placebo cream was applied to the left hand of the subjects who had previously received intravenous naloxone (group 4), no placebo effect was found (Fig. 5B). In fact, no difference in the areas under the curve was found between group 4 and the natural history for the left hand $\left(F_{(1,73)}=0.11 ; p=0.745\right)$, right hand $\left(F_{(1,73)}=0.32 ; p=0572\right)$, left foot $\left(F_{(1,73)}=2.43 ; p=0.123\right)$, and right foot $\left(F_{(1,73)}=0.1 ; p=0.755\right)$. Table 3 shows that no difference was found within group 4 . In addition, the analysis between the two groups 3 and 4 showed a significant difference only on the left hand. Therefore, the specific placebo effect on the left hand, which was induced by the spatial-specific expectation of analgesia on the left hand, was blocked by naloxone.

It should be noted that the subjects who received the lidocaine cream to allow the double-blind design showed a strong analgesic effect only on the left hand in both groups 3 and 4 . In fact, in these subjects, the areas under the curve were the following. In group 3, it was $8.4 \pm 6.3$ on the left hand, $41.3 \pm 15.8$ on the right hand, $44.8 \pm 17.9$ on the left foot, and $39.8 \pm 18.3$ on the right foot. In group 4 , it was $13.3 \pm 9.7$ on the left hand, $45.1 \pm 16.9$ on the right hand, $40.5 \pm 16.7$ on the left foot, and $42.6 \pm 17$ on the right foot. 
A

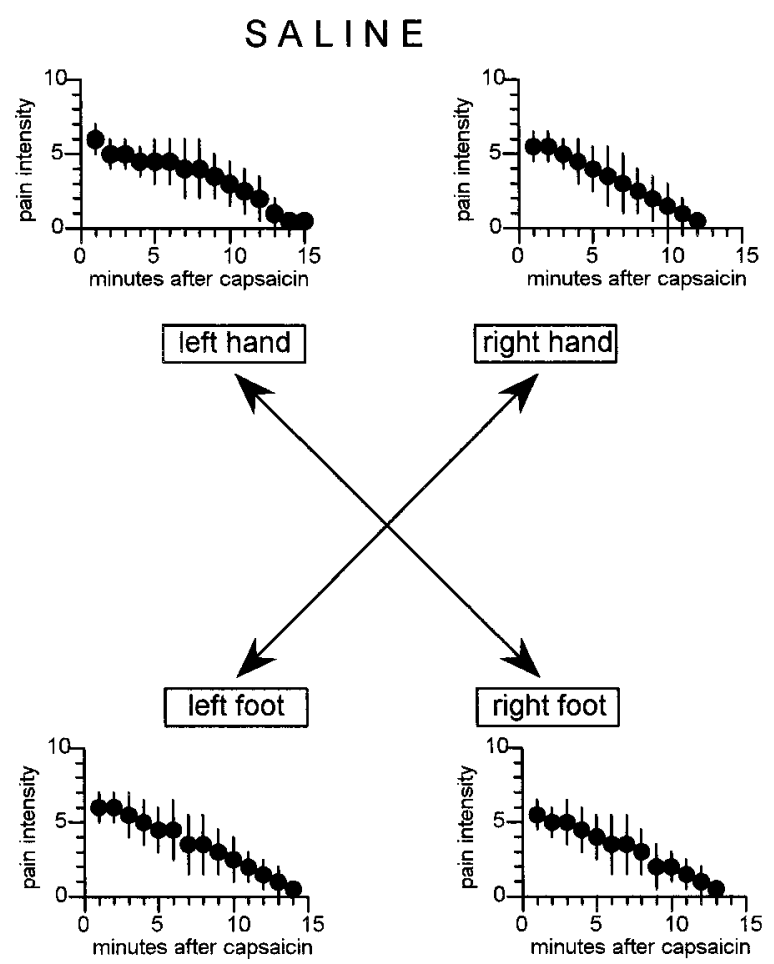

B
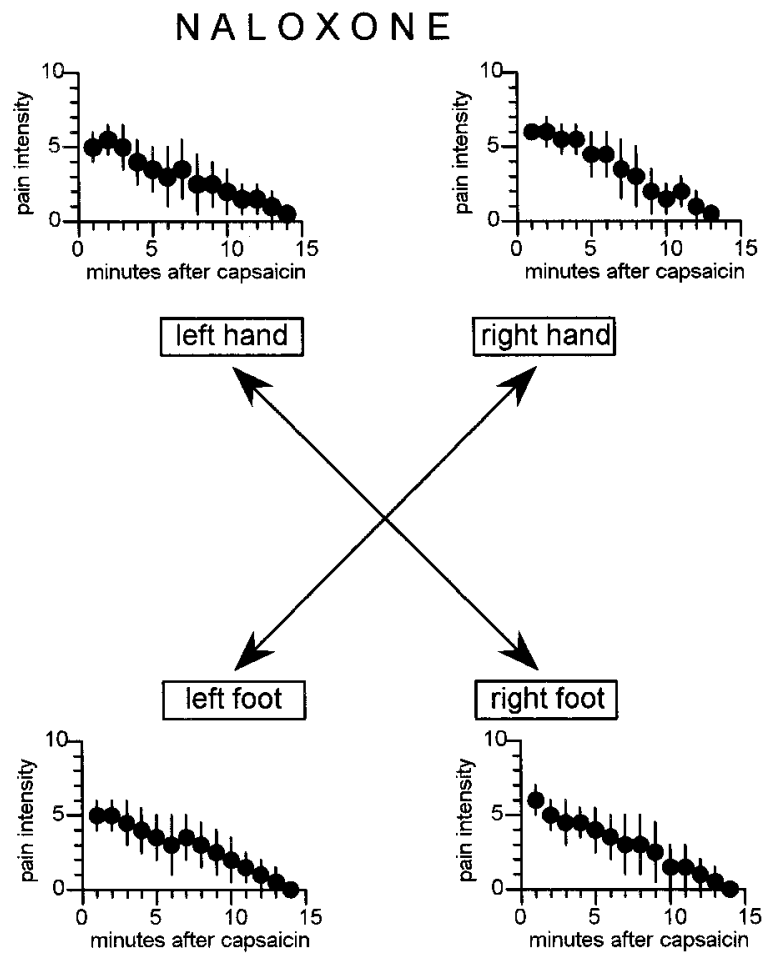

Figure 4. $A$, Natural history of the pain induced by capsaicin on the hands and feet. $B$, Same as in $A$, but these subjects received a hidden injection of naloxone. Note that naloxone did not affect the time course of pain. The statistical analysis of the areas under the curve is shown in Table 2.

Table 2. Comparisons of the areas under the curve within and between groups 1 and 2

\begin{tabular}{llllll} 
& Left hand & Right hand & Left foot & Right foot $^{\prime}$ ANOVA $^{a}$ \\
\hline Group 1 (natural history) & $45.9 \pm 16.1$ & $40.1 \pm 14.7$ & $47.0 \pm 18.2$ & $44.3 \pm 14.3$ & $F_{(3,188)}=1.74 ; p=0.160$ \\
Group 2 (hidden naloxone) & $40.4 \pm 15.7$ & $44.7 \pm 16.6$ & $41.8 \pm 18.8$ & $45.6 \pm 13.9$ & $F_{(3,76)}=0.44 ; p=0.722$ \\
ANOVA $^{b}$ & $F_{(1,66)}=1.67 ;$ & $F_{(1,66)}=1.28 ;$ & $F_{(1,66)}=1.13 ;$ & $F_{(1,66)}=0.12 ;$ \\
& $p=0.201$ & $p=0.262$ & $p=0.292$ & $p=0.732$ \\
\hline
\end{tabular}

${ }^{a}$ Statistical analysis within a single group.

${ }^{b}$ Statistical analysis between the two groups.

\section{Hand- and foot-directed expectancies of analgesia}

If the placebo cream was applied on one hand and one foot, expectation of analgesia was specifically directed to two parts of the body. When the placebo was applied to the right hand and left foot (group 5), we observed a placebo effect only on these two body parts (Fig. $6 A$ ), as shown by the significant difference of the area under the curve with respect to the natural history $\left(F_{(1,70)}=\right.$ $39.0 ; p<0.001$ for the right hand and $F_{(1,70)}=22.05 ; p<0.001$ for the left foot). No difference was found between group 5 and the natural history for both the left hand $\left(F_{(1,70)}=0.01 ; p=\right.$ $0.979)$ and the right foot $\left(F_{(1,70)}=0.41 ; p=0.523\right)$. The ANOVA and the Newman-Keuls test within group 5 showed a significant difference between the treated parts (right hand, left foot) and the untreated parts (left hand, right foot) (Table 4).

In this case also, the previous infusion of naloxone (group 6) blocked completely the two specific placebo effects (Fig. 6B). In fact, the responses to capsaicin, expressed as the area under the curve, of the left and right hand and left and right foot did not differ from the natural history $\left(F_{(1,75)}=2.51 ; p=0.117 ; F_{(1,75)}=\right.$
$0.07 ; p=0.797 ; F_{(1,75)}=0.45 ; p=0.503 ; F_{(1,75)}=0.05 ; p=0.826$, respectively). No difference was found within group 6 , and the analysis between groups 5 and 6 revealed a significant difference only for the right hand and left foot (Table 4). Therefore, in this case also, the specific placebo effects on the right hand and left foot, which were induced by spatial-specific expectancies, were completely blocked by naloxone.

The subjects who received the lidocaine cream to allow the double-blind design showed a strong analgesic effect only on the right hand and left foot in both groups 5 and 6 , as shown by the following areas under the curve. In group 5, the area under the curve was $40.9 \pm 15.9$ on the left hand, $9.1 \pm 7.1$ on the right hand, $11.3 \pm 7.8$ on the left foot, and $42.4 \pm 17.6$ on the right foot. In group 6 , it was $43.8 \pm 18.1$ on the left hand, $12.9 \pm 8.4$ on the right hand, $15.6 \pm 9$ on the left foot, and $39.7 \pm 17.4$ on the right foot.

\section{The effects of placebo on capsaicin-induced allodynia}

The placebo affected not only the time course of capsaicin pain but also the pain thresholds on the cutaneous areas where capsa- 
A

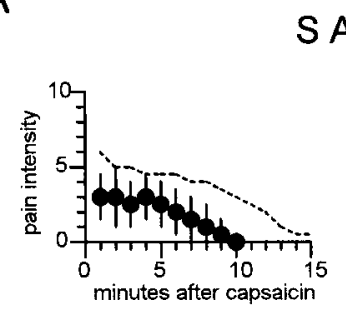

SALINE

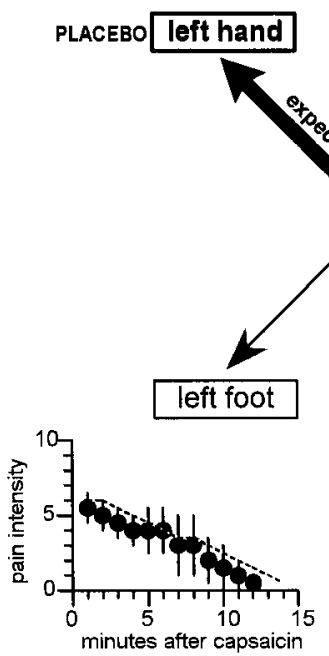

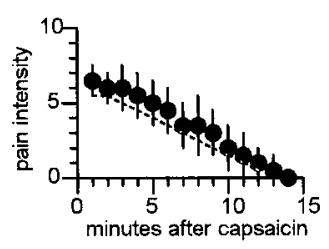

right hand

Figure 5. The effects of the application of a placebo cream on the left hand. The specific expectation of analgesia on the left hand produced a placebo effect only on the left hand and not on the other parts of the body $(A)$. This highly specific placebo effect was completely blocked by naloxone $(B)$. The broken lines show the natural histories of Figure $4 A$. The statistical analysis of the areas under the curve is shown in Table 3.

Table 3. Comparisons of the areas under the curve within and between groups 3 and 4

\begin{tabular}{|c|c|c|c|c|c|}
\hline & Left hand & Right hand & Left foot & Right foot & $\mathrm{ANOVA}^{a}$ \\
\hline \multirow[t]{2}{*}{ Group 3 (placebo on left hand) } & $21.1 \pm 18.3$ & $46.1 \pm 16.1$ & $40.9 \pm 16.8$ & $41.7 \pm 17.0$ & $\begin{array}{c}F_{(3,96)}=10.64 \\
\quad p<0.001\end{array}$ \\
\hline & \multicolumn{4}{|c|}{$\begin{array}{r}-q(96)=7.323 ; p<0.01 \\
-q(96)=5.8 ; p<0.01- \\
-q(96)=6.034 ; p<0.01\end{array}$} & \\
\hline $\begin{array}{l}\text { Group } 4 \text { (placebo on left hand } \\
\text { plus naloxone) }\end{array}$ & $47.2 \pm 17.3$ & $42.1 \pm 14.6$ & $40.5 \pm 15.6$ & $43.2 \pm 15.2$ & \multirow[t]{2}{*}{$\begin{array}{c}F_{(3,104)}=0.89 ; \\
\quad p=0.447\end{array}$} \\
\hline $\mathrm{ANOVA}^{b}$ & $\begin{array}{c}F_{(1,50)}=27.95 \\
\quad p<0.001\end{array}$ & $\begin{array}{c}F_{(1,50)}=0.88 \\
\quad p=0.352\end{array}$ & $\begin{array}{l}F_{(1,50)}=0.01 \\
\quad p=0.929\end{array}$ & $\begin{array}{c}F_{(1,50)}=0.11 \\
\quad p=0.738\end{array}$ & \\
\hline
\end{tabular}

${ }^{a}$ Statistical analysis within a single group.

${ }^{b}$ Statistical analysis between the two groups.

icin had been injected. At 30 min after capsaicin injection, the percentage reduction in pain threshold was much smaller on those parts of the body where the placebo cream had been applied. Figure 7 and Table 5 show that allodynia was significantly less pronounced at $30 \mathrm{~min}$ on the left hand in group 3 (Fig. $7 C$ ) and on the right hand and left foot in group 5 (Fig. $7 E$ ). The Newman-Keuls multiple range test showed that the pain threshold of the left hand in group 3 was higher at 30 min compared with all the other parts of the body (Table 5, $q$ coefficient). Similarly, in group 5, the pain threshold was higher on the right hand and left foot compared with the left hand and right foot. In all the other groups, no significant difference was found at $30 \mathrm{~min}$. When the assessment of allodynia was performed $120 \mathrm{~min}$ after capsaicin injection, no significant difference was present in all
B
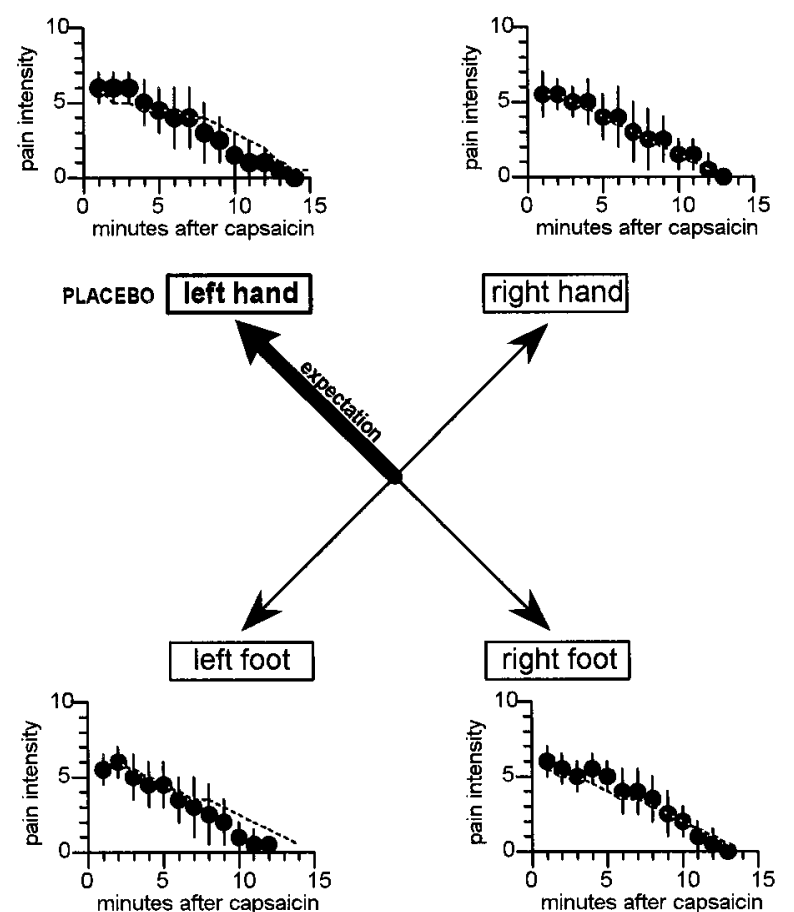
A

\section{SALINE}
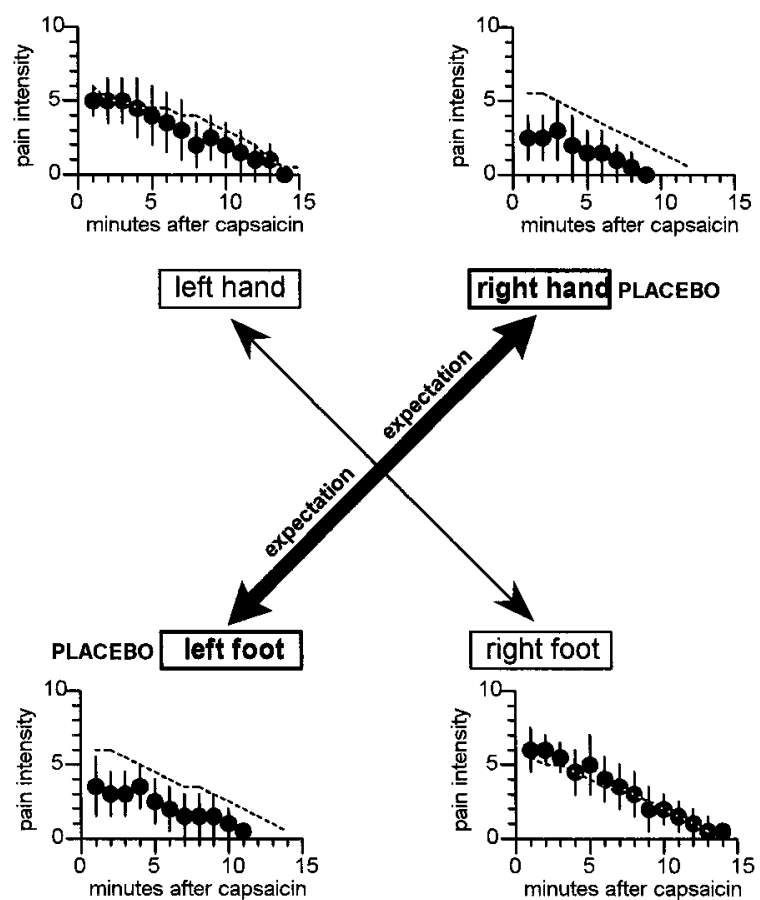

B
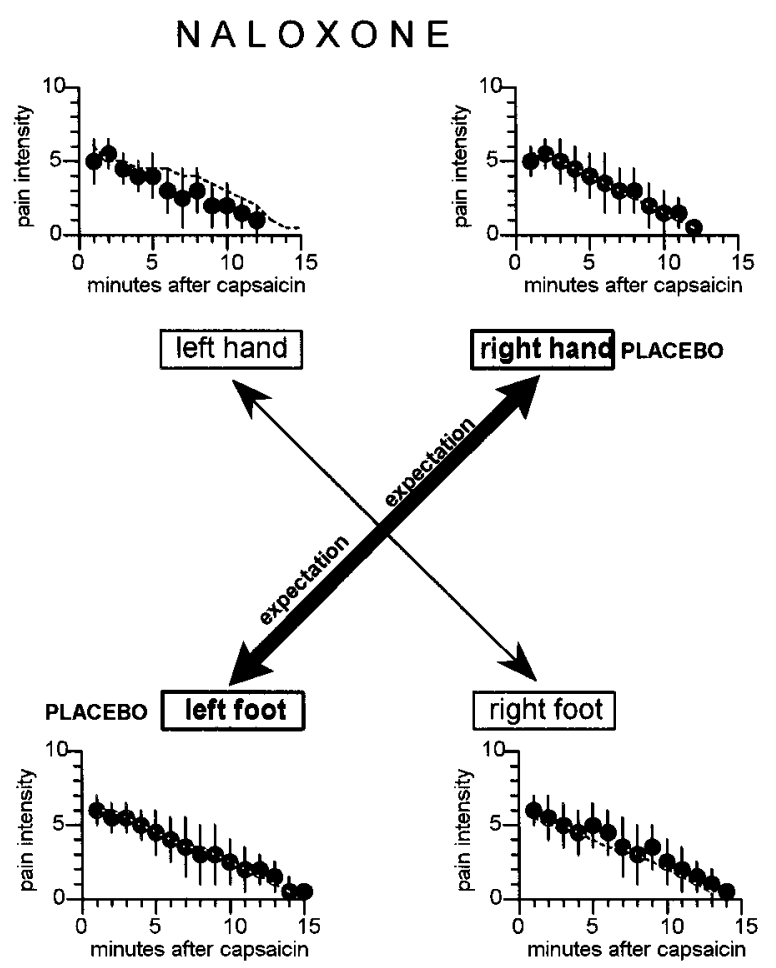

Figure 6. The effects of the application of a placebo cream on the right hand and left foot. The specific expectations of analgesia on the hand and foot produced specific placebo effects on the right hand and left foot, whereas the other two parts of the body were unaffected $(A)$. These specific placebo effects were completely blocked by naloxone $(B)$. The broken lines show the natural histories of Figure $4 A$. The statistical analysis of the areas under the curve is shown in Table 4.

\begin{tabular}{|c|c|c|c|c|c|}
\hline & Left hand & Right hand & Left foot & Right foot & $\mathrm{ANOVA}^{a}$ \\
\hline \multirow[t]{5}{*}{$\begin{array}{l}\text { Group } 5 \text { (placebo on right hand } \\
\text { and left foot) }\end{array}$} & $45.9 \pm 13.8$ & $17.2 \pm 14.6$ & $25.4 \pm 18.8$ & $46.7 \pm 16.2$ & $\begin{array}{l}F_{(3,92)}=20.68 \\
\quad p<0.001\end{array}$ \\
\hline & \multicolumn{2}{|c|}{$\multimap q(92)=8.807 ; p<0.01-$} & & & \\
\hline & \multirow{2}{*}{\multicolumn{4}{|c|}{$q(92)=6.291 ; p<0.01 \longrightarrow$}} & \\
\hline & & $\longrightarrow q(92)=9$ & & & \\
\hline & & & \multicolumn{2}{|c|}{$-q(92)=6.536 ; p<0.01-$} & \\
\hline $\begin{array}{l}\text { Group } 6 \text { (placebo on right hand } \\
\text { and left foot plus naloxone) }\end{array}$ & $39.8 \pm 16.8$ & $41.0 \pm 15.1$ & $44.2 \pm 16.8$ & $43.5 \pm 17.1$ & \multirow[t]{2}{*}{$\begin{array}{l}F_{(3,112)}=0.46 \\
\quad p=0.712\end{array}$} \\
\hline $\mathrm{ANOVA}^{b}$ & $\begin{array}{c}F_{(1,51)}=2.03 \\
\quad p=0.160\end{array}$ & $\begin{array}{l}F_{(1,51)}=33.61 \\
\quad p<0.001\end{array}$ & $\begin{array}{l}F_{(1,51)}=14.77 \\
\quad p<0.001\end{array}$ & $\begin{array}{c}F_{(1,51)}=0.48 \\
\quad p=0.491\end{array}$ & \\
\hline
\end{tabular}

${ }^{a}$ Statistical analysis within a single group.

${ }^{b}$ Statistical analysis between the two groups.

\section{DISCUSSION}

Two important findings emerge from this study. First, a placebo response occurs only on the part of the body where expectation is directed, indicating that the underlying mechanisms do not affect the entire body. Second, this highly specific placebo response is mediated by opioid systems, indicating that the activated endogenous opioids do not act throughout the nervous system but only on those neural circuits linking specific expectations to specific placebo responses.

The experimental procedure we used was aimed at assessing capsaicin pain simultaneously on four parts of the body. To do this, we performed several pilot experiments and found that the subcutaneous injection of capsaicin was an adequate pain stimu- lus for the following reasons. First, the pain burning sensation was very localized, so that a shift of attention from one part of the body to another was easily accomplished. Second, it lasted 12-15 min, which represents a necessary condition to elicit a placebo response. In fact, brief stimuli (few seconds) are usually unaffected by placebos (Price, 1988; Price and Fields, 1997). Third, we used a small dose compared with previous studies (e.g., LaMotte et al., 1991), so that a strong but bearable burning sensation was produced. Fourth, the inf usion pump permitted the simultaneous injection of capsaicin. In contrast, in pilot experiments, we found that it is very difficult to produce simultaneous ischemic pain on hands and feet by means of the tourniquet technique. As far as the judgments of pain are concerned, it 

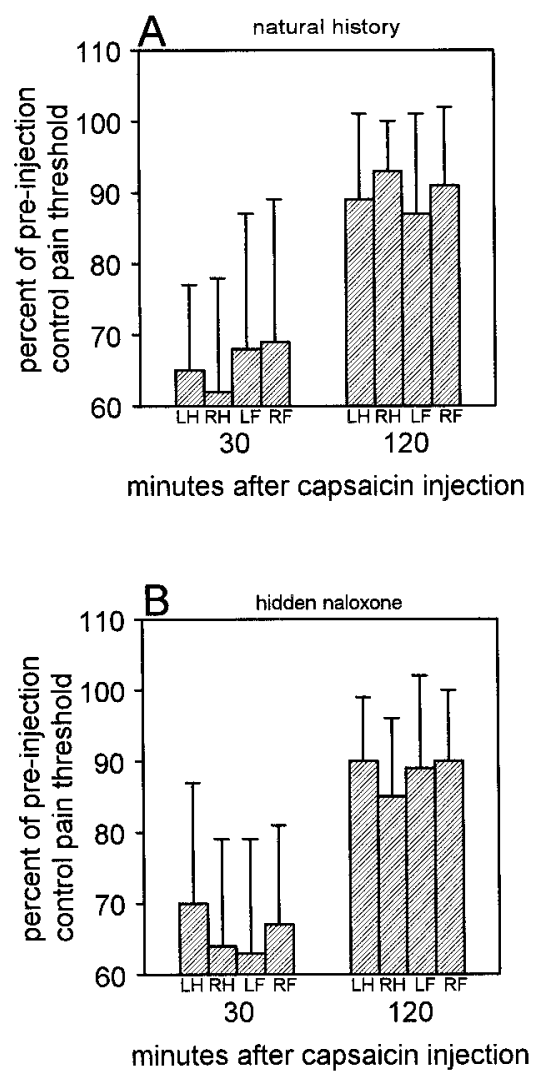
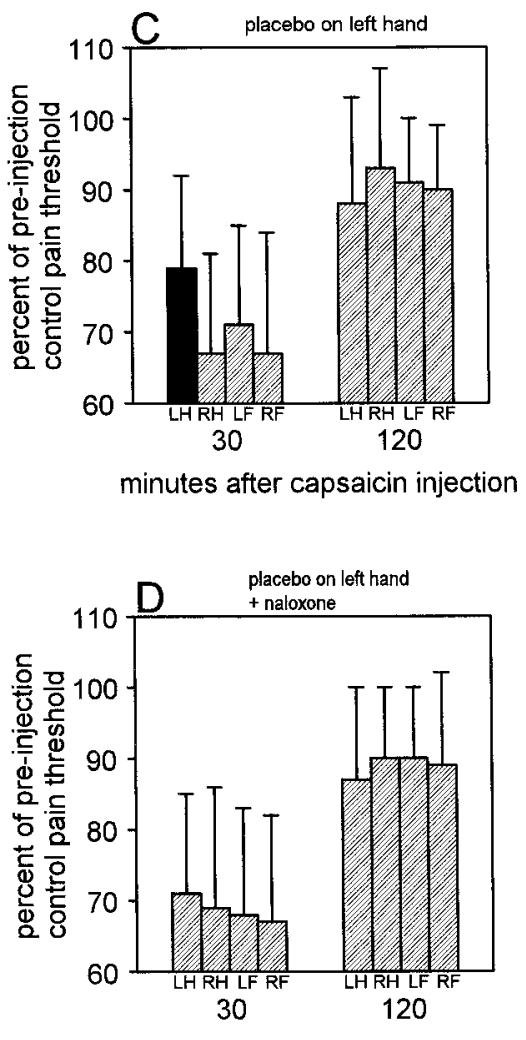

minutes after capsaicin injection
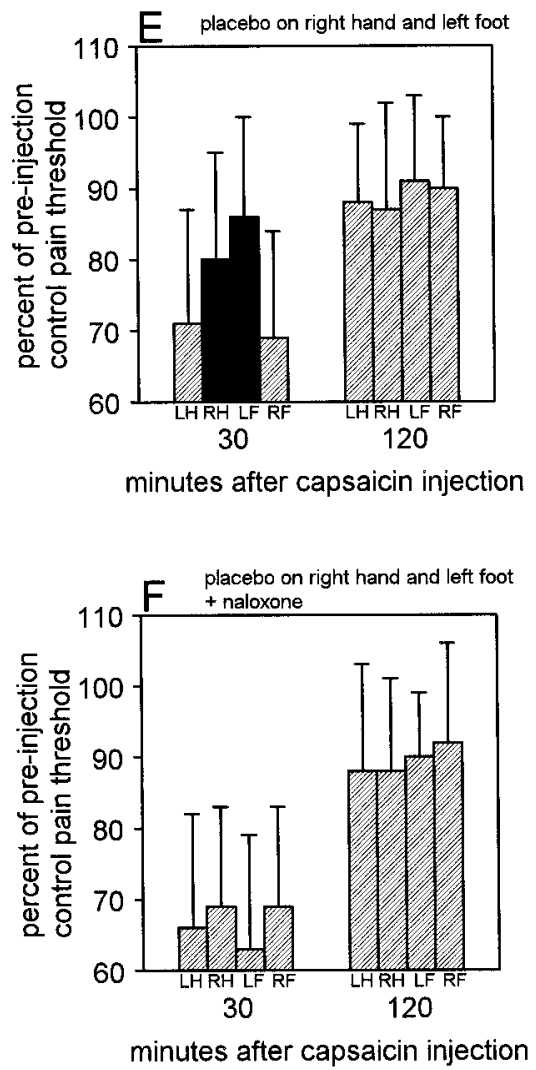

Figure 7. Percent of preinjection control pain threshold at 30 and $120 \mathrm{~min}$ after capsaicin injection in all experimental groups. No significant differences can be observed in $A, B, D$, and $F$. In contrast, in $C$ and $E$, the black columns represent those parts of the body where the placebo cream was applied and where pain thresholds result to be significantly higher compared with the other parts of the body. $L H$, Left hand; $R H$, right hand; $L F$, left foot; $R F$, right foot.

should be pointed out that, for each minute after capsaicin injection, the subjects judged the four pain sensations in $21 \mathrm{sec}$, thus allowing almost concurrent pain ratings on four different body parts. Despite this relatively short period of time, a $7 \mathrm{sec}$ interval was allowed for each pain judgment, and, usually, most of the subjects reported their pain scores 3-4 sec after the electric signal. That this procedure is reliable is demonstrated by the consistency of the pain time course on hands and feet in the natural history group. These data were also confirmed by the assessment of the pain thresholds and allodynia, which clearly show a spatial-specific placebo analgesic effect. In this regard, it is worth emphasizing that, to our knowledge, this is the first study showing a placebo effect on allodynia.

Taking into account these methodological considerations, our data are in agreement with the findings by Montgomery and Kirsch (1996), who showed that some types of local placebo responses cannot be explained by mechanisms affecting the entire body. To reconcile their results with the opioid-mediated placebo effect, Montgomery and Kirsch (1996) and Kirsch (1997) hypothesized that the activation of endogenous opioid systems might occur when placebos are presented as general analgesics, not when placebos are presented as local analgesics. We would like to point out that our study was initially conceived on the basis of this hypothesis, which suggests that different verbal instructions either do or do not activate endogenous opioid systems. Very interestingly, we found that the very specific placebo effects, described by Montgomery and Kirsch (1996) and analyzed in detail in the present study, are mediated by endogenous opioids, indicating that opioid systems can be activated by both general and local placebos.
How can a local and specific placebo response be explained in terms of endogenous opioid activation? First of all, we have to consider that a placebo response may be a result of either cognitive or conditioning mechanisms or both, although other theories have been proposed (for review, see Wall, 1994; Benedetti and Amanzio, 1997). In the first case, expectations and beliefs of analgesia interact with opioid systems to influence the pain pathways (Fields and Price, 1997; Price and Fields, 1997; Amanzio and Benedetti, 1999). In the second case, the placebo response is a learning phenomenon in which previous experience with analgesics and analgesia plays a crucial role (Batterman and Lower, 1968; Laska and Sunshine, 1973; Wickramasekera, 1985; Voudouris et al., 1989, 1990; Benedetti et al., 1998; Amanzio and Benedetti, 1999). However, it is worth stressing that it is not always easy to differentiate between expectation and conditioning. For example, Montgomery and Kirsch (1997) showed that placebo analgesia can result from conditioning but is actually mediated by expectancy. In other words, conditioning leads to the expectation that a given event will follow another event, and this occurs on the basis of the information that the conditioned stimulus provides about the unconditioned stimulus (Rescorla, 1988; Montgomery and Kirsch, 1997). However, a very recent study shows that a real conditioning may occur via opioid receptors in placebo respiratory depression, a situation in which expectancy appears not to be involved (Benedetti et al., 1999). On the basis of these considerations, it is likely that both cognitive and conditioning mechanisms are involved in placebo analgesia in different psychological states and in different circumstances.

The present study adds one more psychological parameter to 


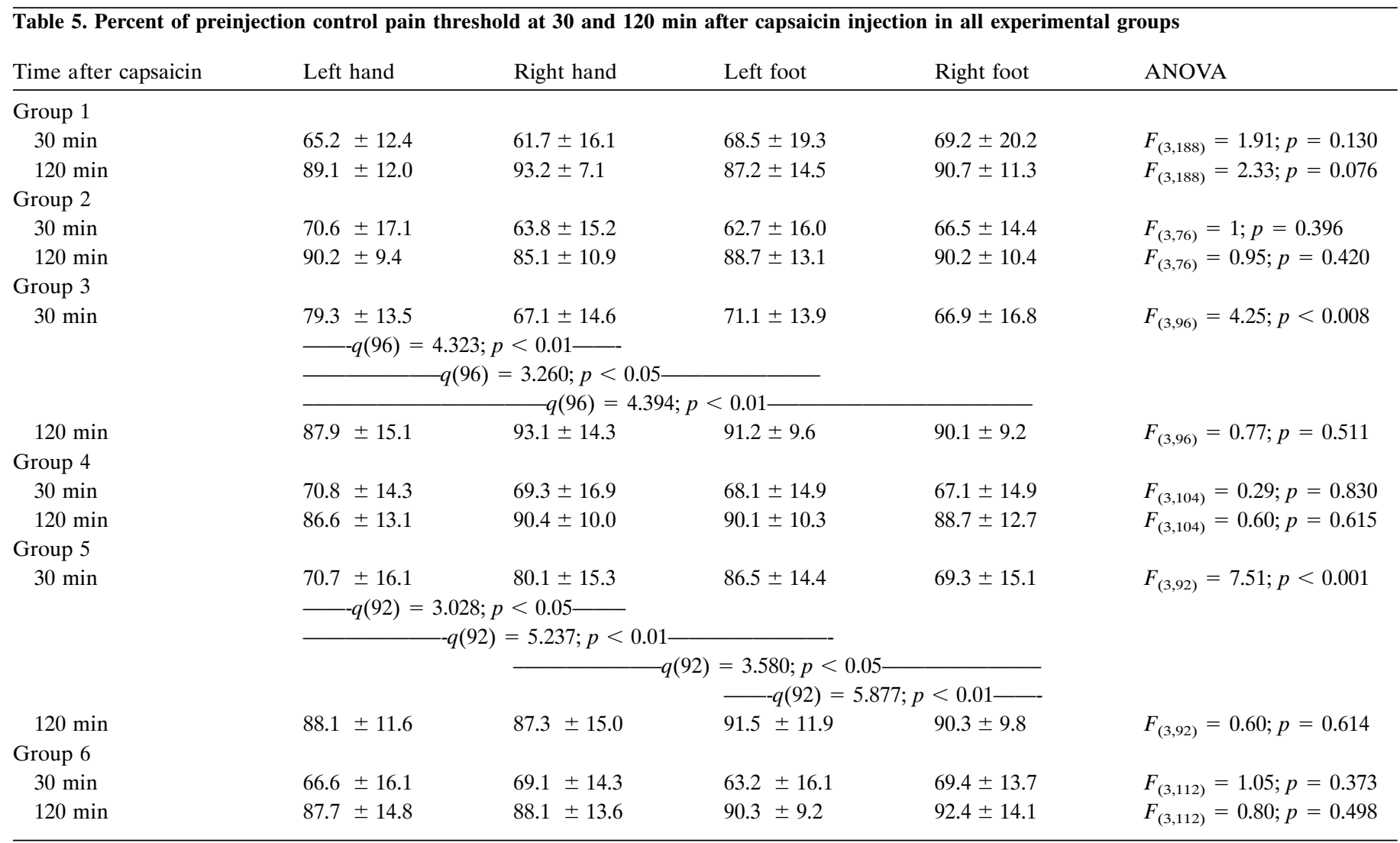

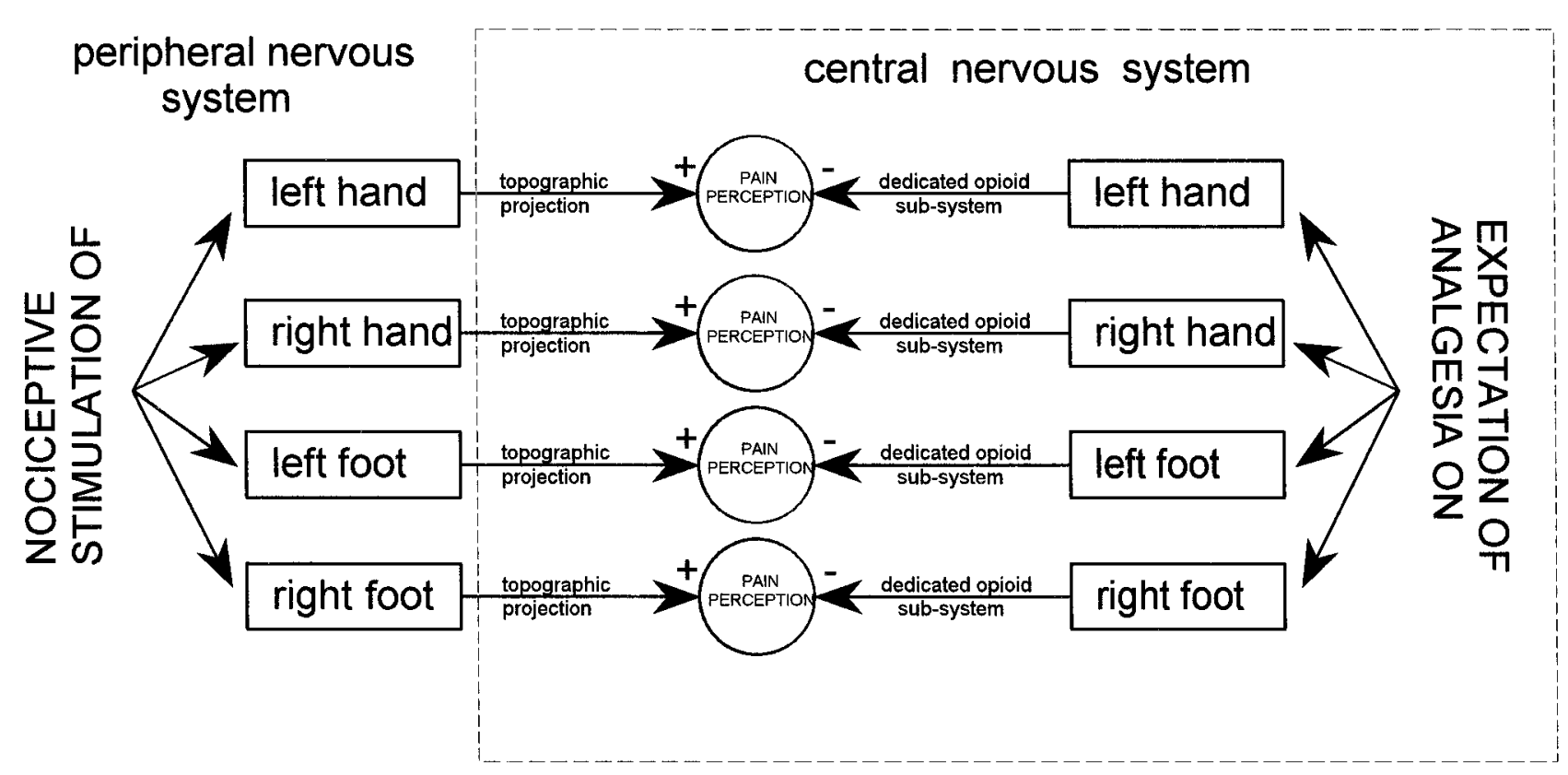

Figure 8. Model explaining the findings of the present study. Spatial-specific expectations of analgesia produce opioid-mediated placebo effects only on those parts where expectations are directed. To do this, specific expectations activate specific opioid subsystems which, in turn, interact with specific topographic representations of the body. This model implies that placebo-activated endogenous opioids are not released throughout the nervous system but are arranged in a highly organized network, linking expectancies, attention, and body schema. +, Excitation; -, inhibition.

the placebo effect, which is the spatially directed expectancy. Although a previous conditioning with analgesics cannot be ruled out (several subjects had a previous experience with analgesics), the spatial dimension of expectation warrants that a cognitive component (i.e., spatial attention) must be present. This spatial attention (or spatially directed expectation) appears to be crucial for the activation of specific opioid systems. These findings lead to some important conclusions. First, if expectation is directed to a 
part of the body, placebo analgesia occurs only on that part, as already demonstrated by Montgomery and Kirsch (1996). Second, this spatially directed expectation implies that attentional mechanisms must be involved. Third, the internal representation of the body (body schema), with its organization and topography, must be the target of the expectancy-mediated placebo analgesic effect. Fourth, all the observed specific placebo effects are mediated by endogenous opioids. Fifth, the effects of these opioids are specifically directed to those neural representations of the body, which are the focus (attention) of the expectations.

Reasoning in this way, a nonspecific release of endogenous opioids throughout the nervous system cannot explain the findings of our study. Rather, a highly organized network of opioid pathways and/or receptors appears to link expectation, attention, and body schema, according to the model shown in Figure 8. Expectation of analgesia can be directed, via attentional mechanisms, to different spatial loci of the body. This complex network activates specific opioid subsystems which, in turn, act only on those parts of the body that are the targets of expectation. Although this model is necessarily speculative, the basic underlying principle appears to be the topographic link between spatially directed expectancies and opioid systems. In other words, spatialspecific expectations have their own organization that is maintained at the level of the endogenous opioid systems.

Modulation of pain by different psychological states is widely recognized, and many studies have focused their attention on brainstem mechanisms and related descending inhibitory control (Mayer and Price, 1976; Basbaum and Fields, 1978, 1984; Watkins and Mayer, 1982; Fields and Price, 1997). For example, Fields (1992), Fields and Basbaum (1994), and Fields and Price (1997) emphasized the role of psychological factors, such as expectation, attention, and arousal, in the modulation of periaqueductal gray and the rostral ventromedial medulla. Most interestingly, Soper and Melzack (1982) showed a somatotopic organization of the periaqueductal gray in rodents, such that the stimulation of different loci produced analgesia in different cutaneous areas. This indicates a high level of anatomical and functional organization in the periaqueductal gray (Bandler and Shipley, 1994). All these pain modulatory networks are affected by opioid peptides, indicating that endogenous opioid systems play an essential role in the organization of both periaqueductal gray and rostral ventromedial medulla (Fields and Basbaum, 1994).

Although the present study cannot demonstrate the involvement of these neural circuits in spatial-specific placebo analgesia, it certainly suggests a topographic relationship between expectancies and opioid systems, occurring for example via the somatotopic organization of the periaqueductal gray. We feel that this finding will help us to understand not only the mechanisms of pain and the organization of the opioid systems but also the interactions between complex mental activities and neurochemical systems.

\section{REFERENCES}

Amanzio M, Benedetti F (1999) Neuropharmacological dissection of placebo analgesia: expectation-activated opioid systems versus conditioning-activated specific sub-systems. J Neurosci 19:484-494.

Bandler R, Shipley MT (1994) Columnar organization in the midbrain periaqueductal gray: modules for emotional expression? Trends Neurosci 9:379-388.

Basbaum AI, Fields HL (1978) Endogenous pain control mechanisms: review and hypothesis. Ann Neurol 4:451-462.

Basbaum AI, Fields HL (1984) Endogenous pain control systems: brainstem spinal pathways and endorphin circuitry. Annu Rev Neurosci 7:309-338.
Batterman RC, Lower WR (1968) Placebo responsiveness-influence of previous therapy. Curr Ther Res 10:136-143.

Benedetti F (1996) The opposite effects of the opiate antagonist naloxone and the cholecystokinin antagonist proglumide on placebo analgesia. Pain 64:535-543.

Benedetti F, Amanzio M (1997) The neurobiology of placebo analgesia: from endogenous opioids to cholecystokinin. Prog Neurobiol 52:109-125.

Benedetti F, Amanzio M, Maggi G (1995) Potentiation of placebo analgesia by proglumide. Lancet 346:1231.

Benedetti F, Amanzio M, Baldi S, Casadio C, Cavallo A, Mancuso M, Ruffini E, Oliaro A, Maggi G (1998) The specific effects of prior opioid exposure on placebo analgesia and placebo respiratory depression. Pain 75:313-319.

Benedetti F, Amanzio M, Baldi S, Casadio C, Maggi G (1999) Inducing placebo respiratory depressant responses in humans via opioid receptors. Eur J Neurosci 11:625-631.

Fields HL (1992) Is there a facilitating component to central pain modulation? Am Pain Soc J 1:139-141.

Fields HL, Basbaum AI (1994) Central nervous sytem mechanisms of pain modulation. In: Textbook of pain (Wall PD, Melzack R, eds), pp 243-257. Edinburgh: Churchill Livingstone.

Fields HL, Levine JD (1984) Placebo analgesia—a role for endorphins? Trends Neurosci 7:271-273.

Fields HL, Price DD (1997) Toward a neurobiology of placebo analgesia. In: The placebo effect: an interdisciplinary exploration (Harrington A, ed), pp 93-116. Cambridge, MA: Harvard UP.

Gracely RH, Dubner R, Wolskee PJ, Deeter WR (1983) Placebo and naloxone can alter postsurgical pain by separate mechanisms. Nature 306:264-265.

Grevert P, Albert LH, Goldstein A (1983) Partial antagonism of placebo analgesia by naloxone. Pain 16:129-143.

Kirsch I (1997) Specifying nonspecifics: psychological mechanisms of placebo effects. In: The placebo effect: an interdisciplinary exploration (Harrington A, ed), pp 166-186. Cambridge, MA: Harvard UP.

LaMotte RH, Shain CN, Simone DA, Tsai E-FP (1991) Neurogenic hyperalgesia: psychophysical studies of underlying mechanisms. J Neurophysiol 66:190-211.

Laska E, Sunshine A (1973) Anticipation of analgesia: a placebo effect. Headache 13:1-11.

Levine JD, Gordon NC (1984) Influence of the method of drug administration on analgesic response. Nature 312:755-756.

Levine JD, Gordon NC, Fields HL (1978) The mechanism of placebo analgesia. Lancet 2:654-657.

Mayer DJ, Price DD (1976) Central nervous system mechanisms of analgesia. Pain 2:379-404.

Montgomery GH, Kirsch I (1996) Mechanisms of placebo pain reduction: an empirical investigation. Psychol Sci 7:174-176.

Montgomery GH, Kirsch I (1997) Classical conditioning and the placebo effect. Pain 72:107-113.

Price DD (1988) Psychological and neural mechanisms of pain. New York: Raven.

Price DD, Fields HL (1997) The contribution of desire and expectation to placebo analgesia: implications for new research strategies. In: The placebo effect: an interdisciplinary exploration (Harrington A, ed), pp 117-137. Cambridge, MA: Harvard UP.

Rescorla RA (1988) Pavlovian conditioning: it is not what you think it is. Am Psychol 43:151-160.

Soper WY, Melzack R (1982) Stimulation-produced analgesia: evidence for somatotopic organization in the midbrain. Brain Res 251:301-311.

Voudouris NJ, Peck CL, Coleman G (1989) Conditioned response models of placebo phenomena: further support. Pain 38:109-116.

Voudouris NJ, Peck CL, Coleman G (1990) The role of conditioning and verbal expectancy in the placebo response. Pain 43:121-128.

Wall PD (1994) The placebo and the placebo response. In: Textbook of pain (Wall PD, Melzack R, eds), pp 1297-1308. Edinburgh: Churchill Livingstone.

Watkins LR, Mayer DJ (1982) Organization of endogenous opiate and nonopiate pain control systems. Science 216:1185-1192.

Wickramasekera I (1985) A conditioned response model of the placebo effect: predictions from the model. In: Placebo: theory, research, and mechanisms (White L, Tursky B, Schwartz GE, eds), pp 255-287. New York: Guilford.

Winter CA, Flataker L (1949) Studies of heptazone (6-morpholino-4,4diphenyl-3-heptanone hydrochloride) in comparison with other analgesic drugs. J Pharmacol Exp Ther 98:305-317. 